\title{
INTERPRETAÇÕES PALEOCLIMÁTICAS A PARTIR DA FLORA PLIOCENNICA DE NOVA IORQUE, MARANHÃO, BRASIL
}

\author{
FRANCISCO SANTIAGO \\ Programa de Pós-Graduação em Geociências, Instituto de Geociências, Universidade Estadual de Campinas, Cx.P. 6152, \\ 13083-970, Campinas, SP, Brasil. santiago1403@gmail.com \\ FRESIA RICARDI-BRANCO \\ Departamento de Geologia e Recursos Naturais, Instituto de Geociências, Universidade Estadual de Campinas, Cx.P. 6152, \\ 13083-970, Campinas, SP, Brasil.fresia@ige.unicamp.br
}

\begin{abstract}
Paleoclimatic interpretations from the Nova Iorque Pliocene flora, Maranhão, Brazil. The Nova Iorque fossil flora, located in northeastern Brazil, is the only fossil flora known for the Pliocene of the Nova Iorque Basin. Because the paleobotanical records in the areas populated by the Caatinga vegetation are scarce, this flora is very important to understand the climatic and environmental changes that occurred in northeastern Brazil. The main physiognomic characteristics - margin type and leaf area - of the angiosperm leaves of 26 species of this flora, allowed us to carry out a quantitative estimation of mean annual temperature (MAT) and mean annual precipitation (MAP) for the moment of its sedimentation. The different equations used to estimate the MAT and the MAP are based on univariate methods known as Leaf Margin Analysis and Leaf Area Analysis. The equations used indicate that the Nova Iorque fossil flora developed under a MAT between 26.1 and $26.7^{\circ} \mathrm{C}$ and a MAP between 580 and $833 \mathrm{~mm}$. These values are very similar to those currently registered in the region where the Nova Iorque fossil flora is located; therefore, this flora lived under a climate similar to that of the Caatinga, which is characterized by being warm and semi-arid. Because this fossil flora has developed under conditions similar to those of the current flora, it is possible to infer that this flora represents a precursor of a phytophysiognomy of the Caatinga.
\end{abstract}

Keywords: Pliocene, Caatinga, angiosperm leaves, Leaf Margin Analysis, Leaf Area Analysis, warm semi-arid climate.

RESUMO - A flora fóssil de Nova Iorque, a qual se encontra localizada no Nordeste Brasileiro, e a única conhecida para o Plioceno da Bacia de Nova Iorque. Uma vez que os registros paleobotânicos nas áreas povoadas pela vegetação da Caatinga são escassos, esta flora representa um elo muito importante para a compreensão dos câmbios climáticos e ambientais que ocorreram no Nordeste Brasileiro. As principais características fisionômicas, tipo de margem e área foliar, das folhas de angiospermas de 26 espécies desta flora permitiram estimar quantitativamente a temperatura média anual (TMA) e precipitação média anual (PMA) para o momento de sua sedimentação. As diferentes equações utilizadas para estimar a TMA e a PMA estão baseadas nos métodos univariados conhecidos como Análise da Margem Foliar e Análise da Área Foliar. As equações empregadas nos indicam que a flora fóssil de Nova Iorque se desenvolveu sob uma TMA de 26,1-26,7 ${ }^{\circ} \mathrm{C}$ e uma PMA de 580-833 mm. Estes valores são muito similares aos que apresenta na atualidade a região onde se encontra a flora fóssil de Nova Iorque, portanto, esta flora viveu sob um clima similar ao da Caatinga, o qual se caracteriza por ser quente e semiárido. Devido a que esta flora fóssil se desenvolveu sob condições semelhantes às da flora atual, permite-se inferir que esta flora representa uma precursora de uma fitofisionomia da Caatinga.

Palavras-chave: Plioceno, Caatinga, folhas de angiospermas, Análise da Margem Foliar, Análise da Área Foliar, clima quente e semiárido.

\section{INTRODUÇÃO}

Os registros paleobotânicos do Nordeste Brasileiro indicam para a maior parte do Neógeno o predomínio de um clima úmido e, somente para o final de esse período a incidência de um clima quente e semiárido (Hoorn et al., 2014). Devido à escassez de registros na região da Caatinga (Hoorn et al., 2014), é de especial interesse a flora fóssil de Nova Iorque, a única registrada para o Plioceno da Bacia de Nova Iorque (Santos \& Carvalho, 2009), para a compreensão da evolução da vegetação e do clima no Nordeste Brasileiro (Cristalli, 1997; Hoorn et al., 2014). Esta flora resulta também importante, uma vez que foi sugerido por estudos prévios que evidenciaria a presença de uma precursora de uma fitofisionomia da Mata Atlântica na área onde atualmente está estabelecida a Caatinga, sob influência de um clima úmido (Cristalli, 1997; Cristalli \& Bernardes-de-Oliveira, 1998; Garcia et al., 2007; Ricardi-Branco \& Fanton, 2007; Hoorn et al., 2014). Esta afirmação está baseada na análise de sua composição florística (Cristalli \& Bernardes-deOliveira, 1998; Ricardi-Branco \& Fanton, 2007; Hoorn et al., 
2014), e das características fisionômicas do material foliar, especificamente o tipo de margem e ápice (Cristalli, 1997; Cristalli \& Bernardes-de-Oliveira, 1998; Hoorn et al., 2014).

As folhas de angiospermas têm sido amplamente empregadas pelos paleobotânicos para a estimativa da temperatura média anual (TMA) e a precipitação média anual (PMA) (Greenwood, 2007; Peppe et al., 2011; Royer, 2012; Wright et al., 2017), já que existe uma covariância entre a proporção de espécies com margens sem dentes e a TMA, assim como entre o tamanho da folha e a PMA (Royer, 2012). Com base nestas relações foram desenvolvidos os métodos univariados, os quais são conhecidos como a Análise da Margem Foliar (Wilf, 1997) e a Análise da Área Foliar (Wilf et al., 1998), que são os mais utilizados por sua simplicidade para a reconstrução da TMA e da PMA (Greenwood, 2007; Peppe et al., 2011; Royer, 2012).

O presente trabalho tem como objetivo estimar quantitativamente os principais parâmetros climáticos, TMA e PMA, para o momento da sedimentação da flora fóssil de Nova Iorque, considerando a boa preservação da margem e do tamanho das folhas de angiospermas.

\section{ÁREA DE ESTUDO}

A flora fóssil de Nova Iorque se encontra localizada na margem esquerda do Rio Parnaíba, perto da antiga vila de Nova Iorque ( $6^{\circ} 45^{\prime} 56^{\prime \prime} \mathrm{S} / 44^{\circ} 3^{\prime} 0^{\prime \prime} \mathrm{O}$; Figura 1$)$, no sudeste do Estado do Maranhão (Cristalli, 1997; Cristalli \& Bernardesde-Oliveira, 1998). Os depósitos cenozoicos desta área encontram-se atualmente sob as águas da Represa de Boa Esperança (Cristalli, 1997; Cristalli \& Bernardes-de-Oliveira, 1998). Tais depósitos foram alocados em uma unidade litoestratigráfica informal, designada como Camadas Nova Iorque (Borges, 1936). Esta unidade encontra-se sobreposta às rochas permianas da Formação Pedra de Fogo do Grupo Balsas (Cristalli, 1997).

As Camadas Nova Iorque se caracterizam por apresentar em sua base um folhelho de $0,5 \mathrm{~m}$ de espessura com grande abundância de fósseis de peixes e algumas folhas, seguido por um folhelho de $0,3 \mathrm{~m}$ de espessura com grande quantidade de fósseis de folhas e poucos peixes, e um folhelho de $0,8 \mathrm{~m}$ de espessura com grande quantidade de fósseis de peixes de pequeno porte e algumas folhas (Borges, 1936; Figura 2). A parte intermediária caracteriza-se por um conglomerado de 2,5 $\mathrm{m}$ de espessura com seixos regulares bem cimentados, e o topo da seção por ter uma argila arenosa com 3,6 m de espessura (Borges, 1936; Figura 2).

A análise dos microfósseis vegetais, esporos e grãos de pólen, permitiu identificar elementos atribuíveis às famílias Alismataceae (?), Aquifoliaceae (Ilex), Arecaceae, Asteraceae (Ambrosia), Euphorbiaceae (Alchornea), Junglandaceae, Lithraceae (Cuphea), Malpighiaceae, Onagraceae (Jussiaea) e Poaceae (Lima in Cristalli, 1997; Dino et al., 2006). Em contrapartida, a análise dos macrofósseis vegetais, folhas de angiospermas, permitiu identificar elementos associados às famílias Clusiaceae, Ebenaceae (cf. Diospyros/ Lisfocarpa), Fabaceae (cf. Caesalpinia, cf. Canavalia, cf. Cassia, cf. Dalbergia), Lauraceae (cf. Laurophyllum), Malvaceae (Malvaciphyllum), Melastomataceae (Miconial Meriania), Meliaceae (cf. Cedrela), Myrtaceae, Rutaceae (?) e Smilacaceae (cf. Smilax) (Cristalli, 1997; Anzótegui \& Cristalli, 2000; Hoorn et al., 2014). Em estudos prévios, a análise dos macrofósseis vegetais, composição taxonômica, e tipo de margem e ápice, sugerem que esta flora possa representar uma precursora de uma fitofisionomia da Mata Atlântica, a qual se desenvolve sob um clima tropical úmido (Cristalli, 1997; Cristalli \& Bernardes-de-Oliveira, 1998; Ricardi-Branco \& Fanton, 2007; Santos \& Carvalho, 2009; Hoorn et al., 2014), que se caracterizava por apresentar uma TMA mínima de $28^{\circ} \mathrm{C}$ (Cristalli, 1997; Cristalli \& Bernardesde-Oliveira, 1998).

As Camadas Nova Iorque depositaram-se durante o Plioceno (Guimarães, 1964; Lima, 1991; Dino et al., 2006). Esta idade lhe foi atribuída inicialmente a partir da análise das estruturas geomorfológicas da rede de drenagem da região onde se encontravam (Guimarães, 1964), e posteriormente, a partir da análise do conteúdo palinológico de seus estratos, no qual foi possível apreciar a presença conjunta das espécies Echitricolporites mcneillyi Germeraad, Hopping \& Muller, Psilatricolpites divisus Regali, Uesugui \& Santos, e Striasyncolpites zwaardi Germeraad, Hopping \& Muller (Lima, 1991; Dino et al., 2006).

\section{MATERIAL E MÉTODOS}

\section{Flora fóssil}

Neste estudo foi analisado o tipo de margem e tamanho foliar de 26 espécies de angiospermas (Tabela 1). Os dados foram adquiridos das descrições de sua arquitetura foliar, as quais foram tomadas da bibliografia (Cristalli, 1997). Estas espécies foram determinadas a partir da análise de 81 amostras de impressões de folhas de angiospermas (Cristalli, 1997), que foram coletadas durante a exploração dos folhelhos das Camadas Nova Iorque (Melo et al., 2005).

\section{Análise da Margem Foliar}

A Análise da Margem Foliar é um método univariado para estimar a TMA, o qual relaciona a proporção de espécies de dicotiledôneas lenhosas sem dentes de uma flora com a TMA (Wilf, 1997; Kennedy et al., 2014). Em alguns casos também se podem incluir espécies de um gênero de monocotiledôneas (Smilax), por sua similaridade foliar com as folhas de dicotiledôneas (Wilf, 1997). Esta correlação não é globalmente uniforme, devido ao fato que o tipo de margem foliar num conjunto florístico está submetido a restrições filogenéticas e históricas (Kennedy et al., 2014). Por esta razão, é recomendável utilizar uma calibração no contexto de uma história fitogeográfica compartilhada para obter estimativas significativas (Hinojosa et al., 2011). Também se recomenda ter um mínimo de 25-30 espécies para obter resultados confiáveis (Wilf, 1997; Burnham et al., 2005). Este método geralmente apresenta um erro de $\pm 5^{\circ} \mathrm{Ce}$, caso se utilize uma calibração regional apropriada, o erro é aproximadamente de $\pm 2^{\circ} \mathrm{C}$ (Royer, 2012). Atualmente considera-se que este 


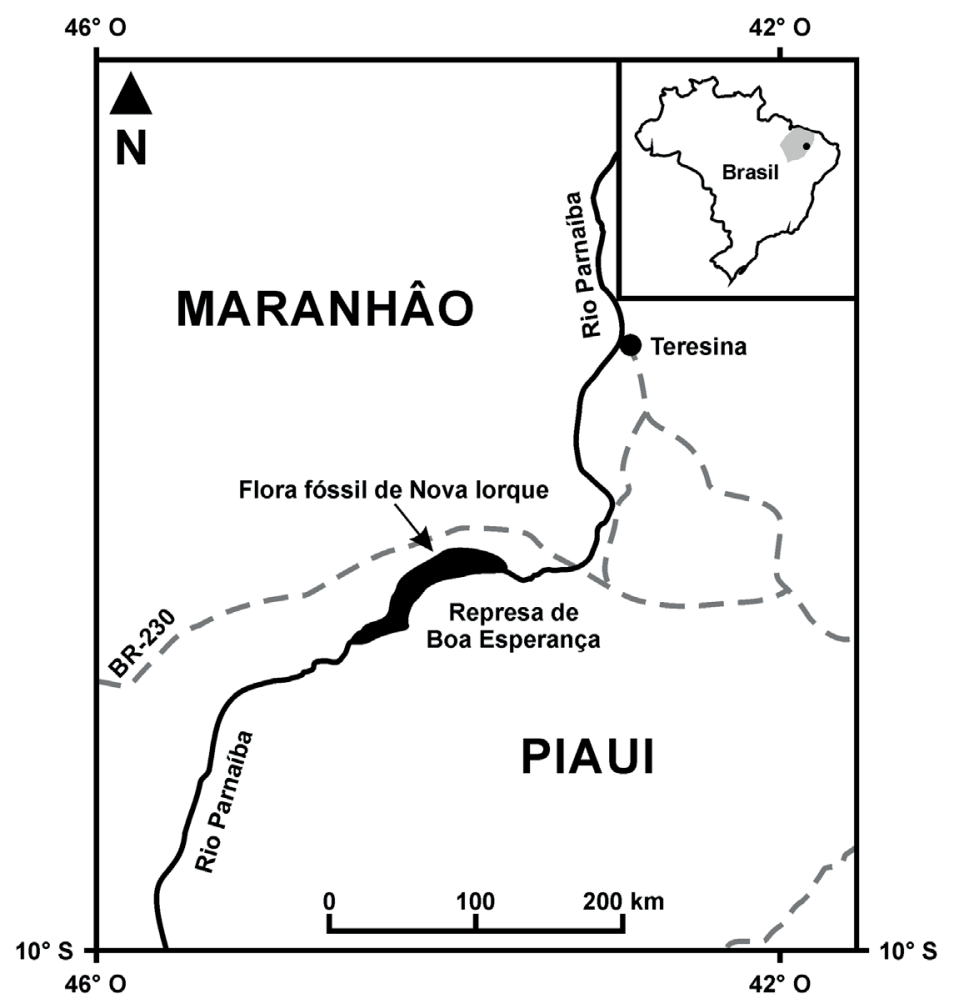

Figura 1. Localização da flora fóssil de Nova Iorque, Camadas Nova Iorque (Anzótegui \& Cristalli, 2000).

Figure 1. Location of the Nova Iorque fossil flora, Nova Iorque Bed (Anzótegui \& Cristalli, 2000).

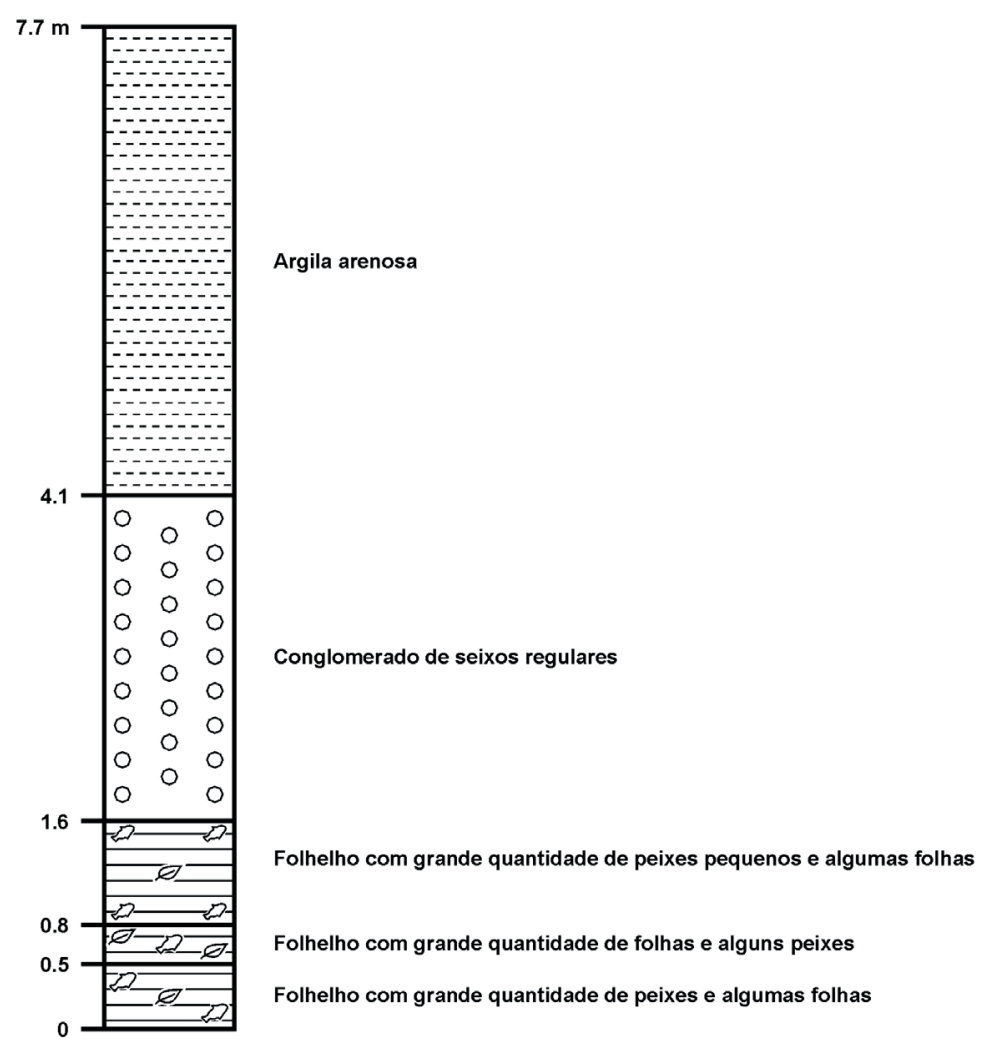

Figura 2. Coluna estratigráfica das Camadas Nova Iorque (Cristalli, 1997).

Figure 2. Stratigraphic column of the Nova Iorque Bed (Cristalli, 1997). 
método sempre subestima a TMA (Peppe et al., 2011), uma vez que as floras fósseis associadas a depósitos fluviais ou lacustres apresentam uma grande proporção de espécies com margem com dentes (Burnham et al., 2001).

Para estimar a TMA foram utilizadas três equações (Tabela 2). A primeira equação foi derivada de um conjunto de dados do hemisfério norte (Wilf, 1997), que pode ser aplicada em floras fósseis sul-americanas, devido à sua precisão para estimar a TMA atual da região tropical da América do Sul (Kowalski, 2002). A segunda e terceira equação foram derivadas de conjuntos de dados de América do Sul (Hinojosa et al., 2011), que é o mais recomendado para a estimar a TMA de floras fósseis da região (Kowalski, 2002; Hinojosa et al., 2011).

\section{Análise da Área Foliar}

A Análise da Área Foliar é um método univariado para estimar a PMA, baseado na relação da área foliar média de uma flora com a PMA (Wilf et al., 1998). Recomenda-se ter um mínimo de 25-30 espécies para sua aplicação (Wilf et al.,
1998; Jacobs \& Herendeen, 2004). Os valores obtidos devem ser cuidadosamente interpretados (Wilf et al., 1998; Peppe et al., 2011), já que as estimativas apresentam um erro de \pm 500 mm (Wilf et al., 1998) a $\pm 1000 \mathrm{~mm}$ (Peppe et al., 2011). Isto se deve principalmente ao fato de que as folhas podem refletir algum tipo de déficit hídrico, devido às características da temperatura, do solo e/ou das águas subterrâneas (Royer, 2012). Para estimar a PMA foram utilizadas três equações, as quais se derivaram de diferentes conjuntos de dados (Tabela 2).

\section{RESULTADOS}

Da análise das 26 espécies da flora fóssil de Nova Iorque, temos 25 que apresentam margem sem dentes, o qual é equivalente a $96 \%$ do total. Este valor permitiu estimar a TMA (Tabela 3). As equações utilizadas produziram diferentes valores que vão de 26,1 a $26,7^{\circ} \mathrm{C}$ (Tabela 3). A partir dos dados da área foliar das 26 espécies analisadas determinou-se inicialmente a proporção relativa em cada classe de tamanho

Tabela 1. Tipo de margem e tamanho das folhas de angiospermas de 26 espécies da flora fóssil de Nova Iorque, Camadas Nova Iorque (Cristalli, 1997). Símbolo: a,tamanho segundo Raunkiaer (1934) modificado por Webb (1959).

Table 1. Margin type and size of the angiosperm leaves of 26 species of the Nova Iorque fossil flora, Nova Iorque Bed (Cristalli, 1997). Symbol: a, size according to Raunkiaer (1934) modified by Webb (1959).

\begin{tabular}{|c|c|c|c|c|}
\hline Família & Morfotipo & Espécie & Tipo de margem & Tamanho $^{\mathrm{a}}$ \\
\hline Ebenaceae & FNNY-(Ebenales) 1 & cf. Diospyros sp. & Sem dentes & Microfilo \\
\hline \multirow[t]{5}{*}{ Fabaceae } & FNNY-(Fabales) 1 & & Sem dentes & Nanofilo \\
\hline & FNNY-(Fabales) 2 & & Sem dentes & Nanofilo \\
\hline & FNNY-(Fabales) 3 & & Sem dentes & Nanofilo \\
\hline & FNNY-(Fabales) 4 & & Sem dentes & Nanofilo \\
\hline & FNNY-(Fabales) 5 & & Sem dentes & Nanofilo \\
\hline Lauraceae & FNNY-(Laurales) 1 & cf. Laurophyllum sp. & Sem dentes & Microfilo \\
\hline Malvaceae & FNNY-(Malvales) 1 & & Com dentes & Microfilo \\
\hline \multirow[t]{2}{*}{ Meliaceae } & FNNY-(Sapindales) 1 & cf. Cedrela sp. 1 & Sem dentes & Microfilo \\
\hline & FNNY-(Sapindales) 2 & cf. Cedrela sp. 2 & Sem dentes & Microfilo \\
\hline Smilacaceae & FNNY-(Liliales) 1 & cf. Smilax sp. & Sem dentes & Nanofilo \\
\hline \multirow[t]{15}{*}{ Indeterminada } & FNNY-(Indet.) 1 & & Sem dentes (?) & Microfilo \\
\hline & FNNY-(Indet.) 2 & & Sem dentes (?) & Microfilo \\
\hline & FNNY-(Indet.) 3 & & Sem dentes (?) & Microfilo \\
\hline & FNNY-(Indet.) 4 & & Sem dentes & Microfilo \\
\hline & FNNY-(Indet.) 5 & & Sem dentes & Nanofilo-Microfilo \\
\hline & FNNY-(Indet.) 6 & & Sem dentes (?) & Microfilo \\
\hline & FNNY-(Indet.) 7 & & Sem dentes & Microfilo-Notofilo \\
\hline & FNNY-(Indet.) 8 & & Sem dentes & Microfilo \\
\hline & FNNY-(Indet.) 9 & & Sem dentes & Microfilo \\
\hline & FNNY-(Indet.) 10 & & Sem dentes & Microfilo \\
\hline & FNNY-(Indet.) 11 & & Sem dentes & Microfilo \\
\hline & FNNY-(Indet.) 12 & & Sem dentes & Microfilo \\
\hline & FNNY-(Indet.) 13 & & Sem dentes & Microfilo \\
\hline & FNNY-(Indet.) 14 & & Sem dentes & Nanofilo-Microfilo \\
\hline & FNNY-(Indet.) 15 & & Sem dentes & Microfilo \\
\hline
\end{tabular}


e posteriormente o logaritmo natural da área foliar (Tabela 4). Este valor permitiu estimar a PMA (Tabela 3). As equações utilizadas produziram diferentes valores que vão de 580 a $833 \mathrm{~mm}$ (Tabela 3).

\section{DISCUSSÃO E CONCLUSÕES}

A reconstrução climática do Cenozoico da zona tropical da América do Sul é profundamente afetada pela contínua elevação dos Andes e as mudanças dos padrões climáticos (Kowalski, 2002), e no caso específico do Nordeste Brasileiro, pelo soerguimento do Planalto da Borborema, o que trouxe consigo mudanças climáticas que influenciaram diretamente na distribuição da vegetação (Hoorn et al., 2014). Apesar disto, as principais características fisionômicas das folhas de angiospermas analisadas permitiram estimar a TMA e a PMA (Tabela 3), assim como a reconstrução da vegetação.

A TMA e a PMA obtidas para a flora fóssil de Nova Iorque são muito similares as que atualmente apresenta a área onde ela se encontrava (Tabela 3). Esta área se encontra nos domínios do Bioma Caatinga, o qual se caracteriza por apresentar um clima quente e semiárido (Velloso et al., 2002; Prado, 2003). As análises macro e microfloristicas também sugere que a flora fóssil de Nova Iorque esteve sob este clima no momento de sua sedimentação (Cristalli, 1997), o qual parece ser próprio desta região durante o Plioceno (Garcia et al., 2007; Hoorn et al., 2014).

O clima da Caatinga é de caráter quente e semiárido, com altas temperaturas e precipitações escassas e irregulares (Prado, 2003). A TMA é de 26 a $28^{\circ} \mathrm{C}$ (Nimer, 1972), e a PMA é de 500 a 1000 mm (Nimer, 1972; Prado, 2003). Estes valores são muito similares aos que foram obtidos para a flora fóssil de Nova Iorque (Tabelas 3). Portanto, é possível inferir que esta flora fóssil se desenvolveu sob condições ambientais semelhantes às da Caatinga, as quais se originam no momento que as massas de ar Atlântico-Equatoriais perdem sua umidade, ao serem empurradas pelos ventos alísios contra o Planalto da Borborema (Prado, 2003; Hoorn et al., 2014).

A Caatinga apresenta uma surpreendente diversidade de ambientes, proporcionados por um mosaico de tipos de vegetação, o qual varia dependendo do tipo de solo e da disponibilidade de água (Velloso et al., 2002; Giulietti et al., 2003; Prado, 2003). A vegetação é constituída essencialmente por árvores e arbustos baixos, sendo que muitos apresentam espinhos, microfilia e algumas características xerofíticas (Prado, 2003). Sua estrutura em relação à altura das árvores e à densidade da vegetação é muito variável, esta pode se apresentar em forma de moitas baixas e isoladas até como

Tabela 2. Equações baseadas na Análise da Margem Foliar e Análise da Área Foliar utilizadas para a estimação da temperatura média anual (TMA) e a precipitação média anual (PMA) da flora fóssil de Nova Iorque, Camadas Nova Iorque. Símbolos: a , número de locais; ${ }^{\text {b }}$, coeficiente de determinação; ${ }^{\text {c }}$ erro padrão do modelo; ' , região geográfica onde foram coletadas as amostras; ${ }^{\mathrm{e}}$, proporção de espécies sem dentes; ${ }^{\mathrm{f}}$, Log natural da área foliar calculado de acordo com Wilf et al. (1998): Mln $A=\Sigma a p_{i}$ (ai = sete médias das áreas do log natural das classes de tamanho de Raunkiaer (1934), modificadas pelo Webb (1959), e $p_{i}=$ a proporção de espécies em cada uma das classes de tamanho).

Table 2. Equations based on the Leaf Margin Analysis and Leaf Area Analysis used to estimate mean annual temperature (MAT) and mean annual precipitation (MAP) of the Nova Iorque fossil flora, Nova Iorque Bed. Symbols: a , number of localities; ${ }^{\text {b }}$, coefficient of determination; ${ }^{\text {c }}$, standard error of the model; ${ }^{\text {d, }}$ geographic region where the samples were collected; ${ }^{\mathrm{e}}$, proportion of species untoothed; ${ }^{\mathrm{f}}$, natural logarithm $\mathrm{f}$ the leaf área calculated according to Wilf $e t$ al. (1998): $M \ln A=\sum \mathrm{a} p_{i}\left(a i=\right.$ seven means of the areas of the natural logarithm of the Raunkiaer (1934) size classes , modified by Webb (1959), and $p_{i}=$ the proportion of species in each size class).

\begin{tabular}{|c|c|c|c|c|c|c|}
\hline & Equação & $\mathrm{n}^{\mathrm{a}}$ & $\mathrm{r}^{2 \mathrm{~b}}$ & $\mathrm{EP}^{\mathrm{c}}$ & Região $^{d}$ & Fonte \\
\hline \multirow{3}{*}{ 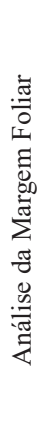 } & (Eq. 1) $\mathrm{TMA}=24,40 E^{\mathrm{e}}+3,25$ & 74 & 0,84 & 2,1 & $\begin{array}{c}\text { América do Norte, } \\
\text { América Central e Japão. }\end{array}$ & Wilf (1997) \\
\hline & (Eq. 2) $\mathrm{TMA}=23,42 E+3,60$ & 44 & 0,48 & 3,5 & $\begin{array}{c}\text { Zona tropical da } \\
\text { América do Sul (Bolívia, } \\
\text { Brasil, Colômbia, } \\
\text { Equador, Guiana, Peru e } \\
\text { Venezuela). }\end{array}$ & Hinojosa et al. (2011) \\
\hline & (Eq. 3) $\mathrm{TMA}=26,03 E+1,31$ & 74 & 0,82 & 2,8 & $\begin{array}{c}\text { América do Sul (Bolívia, } \\
\text { Brasil, Colômbia, Chile, } \\
\text { Equador, Guiana, Peru e } \\
\text { Venezuela). }\end{array}$ & Hinojosa et al. (2011) \\
\hline$\underset{\substack{\pi \\
0}}{\stackrel{7}{0}}$ & (Eq. 4) $\ln P M A=0,548 M \ln A^{\mathrm{f}}+0,768$ & 50 & 0,760 & $0,359 \ln$ & $\begin{array}{c}\text { América do Norte, } \\
\text { América Central, } \\
\text { América do Sul e África. }\end{array}$ & Wilf et al. (1998) \\
\hline 这 & (Eq. 5) $\ln \mathrm{PMA}=2,566+0,309 \mathrm{M} \ln A$ & 42 & 0,734 & -------- & $\begin{array}{c}\text { Zona tropical da África e } \\
\text { Bolívia. }\end{array}$ & $\begin{array}{c}\text { Jacobs \& Herendeen } \\
\text { (2004) }\end{array}$ \\
\hline 莺 & (Eq. 6) $\ln P M A=2,167+0,354 M \ln A$ & 79 & 0,709 & -------- & $\begin{array}{c}\text { América do Norte, } \\
\text { América Central, } \\
\text { América do Sul e África. }\end{array}$ & $\begin{array}{c}\text { Jacobs \& Herendeen } \\
\text { (2004) }\end{array}$ \\
\hline
\end{tabular}


Tabela 3. Estimativa da temperatura média anual (TMA) e a precipitação média anual (PMA) da flora fóssil de Nova Iorque, Camadas Nova Iorque, com base na Análise da Margem Foliar e na Análise da Área Foliar. Símbolos: a , proporção de espécies sem dentes; ' , logaritmo natural da área foliar; ${ }^{\text {, }}$ temperatura media anual e precipitação média anual do Município de Nova Iorque (Rodrigues, 2008).

Table 3. Estimate of the mean annual temperature (MAT) and mean annual precipitation (MAP) of the Nova Iorque fossil flora, Nova Iorque Bed, based on the Leaf Margin Analysis and Leaf Area Analysis. Symbols: ${ }^{\text {a }}$, proportion of species untoothed; ${ }^{\mathbf{b}}$, natural logarithm of leaf area; ${ }^{\mathbf{c}}$, mean annual temperature and mean annual precipitation of the Municipality of Nova Iorque (Rodrigues, 2008).

\begin{tabular}{cccc}
\hline \multicolumn{2}{c}{$\begin{array}{c}\text { Análise da Margem Foliar } \\
\mathrm{n}=0,96^{\mathrm{a}}\end{array}$} & \multicolumn{2}{c}{$\begin{array}{c}\text { Análise da Área Foliar } \\
M \operatorname{lnA}=6,01^{\mathrm{b}}\end{array}$} \\
\hline Equação & $\mathrm{TMA}\left({ }^{\circ} \mathrm{C}\right)$ & Equação & PMA (mm) \\
\hline Eq. 1 & $26,7 \pm 2,1$ & Eq. 4 & $580^{+249}{ }_{-175}$ \\
Eq. 2 & $26,1 \pm 3,5$ & Eq. 5 & 733 ------- \\
Eq. 3 & $26,3 \pm 2,8$ & Eq. 6 & 1110 \\
\hline Atual & 27,2 & Atual & \\
\hline
\end{tabular}

Tabela 4. Dados da área foliar das folhas presentes na flora fóssil de Nova Iorque, Camadas Nova Iorque. Símbolos: a, classes de tamanho segundo Raunkiaer (1934) modificadas por Webb (1959); ${ }^{\mathbf{b}}$, proporções relativas; ${ }^{\mathbf{c}}$, média da área log natural para cada classe de tamanho. ${ }^{\mathbf{}}$, log natural da área foliar calculado de acordo com Wilf et al. (1998), onde $M \ln A=\Sigma a_{i} p_{i}$.

Table 4. Leaf area data of the leaves present in the Nova Iorque fossil flora, Nova Iorque Bed. Symbols: a, size classes according to Raunkiaer (1934) modified by Webb (1959); ${ }^{\text {, }}$, relative ratios; ${ }^{\mathbf{c}}$, mean of the natural logarithm area for each size class; ${ }^{\mathbf{d}}$, natural logarithm of the leaf area calculated according to Wilf et al. (1998), where $M \ln A=\Sigma a p_{i}$.

\begin{tabular}{|c|c|c|c|c|}
\hline Classe de tamanho $^{\mathrm{a}}$ & Faixa de classe de tamanho $\left(\mathrm{cm}^{2}\right)$ & $p_{i}$ Classes de tamanho $^{\mathrm{b}}$ & $a_{i}^{\mathrm{c}}$ & $M \ln A^{\mathrm{d}}\left(a p_{i}\right)$ \\
\hline Leptofilo & $\leq 0.25$ & 0,00 & 2,12 & 0,00 \\
\hline Nanofilo & $>0,25-2,25$ & 0,25 & 4,32 & 1,08 \\
\hline Microfilo & $2,25-20,25$ & 0,72 & 6,51 & 4,69 \\
\hline Notofilo & $20,25-45,00$ & 0,03 & 8,01 & 0,24 \\
\hline Mesofilo & $45,00-182,25$ & 0,00 & 9,11 & 0,00 \\
\hline Macrofilo & $182,25-1640,20$ & 0,00 & 10,90 & 0,00 \\
\hline \multirow[t]{2}{*}{ Megafilo } & $>1640,20$ & 0,00 & 13,10 & 0,00 \\
\hline & & & $\sum a p_{i}$ & 6,01 \\
\hline
\end{tabular}

uma floresta fechada (Velloso et al., 2002; Giulietti et al., 2003; Prado, 2003). Dado que as fitofisionomias da Caatinga são muito variáveis, estas têm sido generalizadas em poucos tipos, tais como arbórea-arbustiva aberta, arbórea-arbustiva fechada e arbórea fechada (Prado, 2003). A Caatinga também se caracteriza pelo seu alto grau de endemismo florístico (Giulietti et al., 2003; Prado, 2003), embora a metade de seu estoque tenha sido derivado das florestas tropicais da Mata Atlântica (Rizzini, 1963, 1979). Devido ao seu alto grau de endemismo florístico, a vegetação da Caatinga tem apresentado uma distribuição semelhante à atual por um longo período de tempo (Prado, 2003). Contudo, ela já deveria estar firmemente estabelecida no Plioceno (Ab'Sáber, 1974; Pennington et al., 2004), onde sofreu uma primeira expansão (Werneck et al., 2011).

Nas terras mais baixas da Caatinga se apresentam um conjunto de hábitats frágeis ao redor das lagoas ou áreas úmidas temporárias, os quais são refúgios de muitas espécies aquáticas, vegetais e animais, que desempenham um papel fundamental na sobrevivência de muitas espécies de peixes, aves e mamíferos, que completam seu ciclo de vida associados a esses ambientes (Giulietti et al., 2003). A análise tafonômica da flora fóssil de Nova Iorque sugere que ela foi depositada num ambiente lacustre com características similares (Cristalli, 1997; Cristalli \& Bernardes-de-Oliveira, 1998).

Em estudos prévios, a análise da composição taxonômica da flora fóssil de Nova Iorque, assim como de suas principais características fisionômicas, tipo de margem e ápice, sugeriram que esta flora poderia ser uma precursora de uma fitofisionomia da Mata Atlântica (Cristalli, 1997; Cristalli \& Bernardes-de-Oliveira, 1998; Ricardi-Branco \& Fanton, 2007; Hoorn et al., 2014). Contudo, o atual estudo sugere que a flora fóssil de Nova Iorque teria mais relações com as fitofisionomias da Caatinga do que com as da Mata Atlântica, embora tivesse um caráter sempre verde, com um dossel parcialmente fechado e com a presença de no máximo dois estratos, um arbustivo e outro arbóreo (Cristalli, 1997; Cristalli \& Bernardes-de-Oliveira, 1998; Ricardi-Branco \& Fanton, 2007; Hoorn et al., 2014). Isto se deve ao fato de que a Caatinga durante o Plioceno se expandiu, como resultado das mudanças climáticas, o que permitiu uma conexão entre a Floresta Amazônica e a Mata Atlântica (Batalha-Filho et al., 2013).

Portanto, é possível concluir que a flora fóssil de Nova Iorque, se desenvolveu sob um clima quente e semiárido, que se caracterizava por apresentar uma TMA de $26,1-26,7^{\circ} \mathrm{C}$ e 
uma PMA de 580-833 mm. Devido a que estas características encontradas na flora fóssil de Nova Iorque são similares às da região atual da Caatinga, permite-se inferir que esta flora fóssil representa uma precursora de uma fitofisionomia da mesma.

\section{AGRADECIMENTOS}

Os autores agradecem o apoio financeiro da Fundação de Amparo à Pesquisa do Estado de São Paulo (FAPESP; projeto Studies of Modern and fossil bioclast accumulations related to continental and coastal environments - 2016/209270), a Coordenação de Aperfeiçoamento de Pessoal de Nível Superior (CAPES) e ao Conselho Nacional de Desenvolvimento Científico e Tecnológico (CNPQ) pelas bolsas concedidas. Os autores também expressam seus agradecimentos aos avaliadores, A. Iglesias e L.M. Anzótegui, e ao editor, A.M. Ribeiro, pelas valiosas sugestões que ajudaram a melhorar o manuscrito.

\section{REFERÊNCIAS}

Ab'Sáber, A.N. 1974. O dominio morfoclimático semi-árido das Caatingas brasileiras. Geomorfologia, 43:1-39.

Anzótegui, L.M. \& Cristalli, P. 2000. Primer registro de hojas de Malvaceae en el Neógeno de Argentina y Brasil. Ameghiniana, 37:169-180.

Batalha-Filho, H.; Fjeldså, J.; Fabre, P.H. \& Miyaki, C.Y. 2013. Connections between the Atlantic and the Amazonian forest avifaunas represent distinct historical events. Journal of Ornithology, 154:41-50. doi:10.1007/s10336-012-0866-7

Borges, J. 1936. Atividades do sub-assistente José Alfredo Borges. Relatório annual do Diretor. Rio de Janeiro, Departamento Nacional da Produção Mineral, 97 p.

Burnham, R.J.; Ellis, B. \& Johnson, K.R. 2005. Modern tropical forest taphonomy: does high biodiversity affect paleoclimatic interpretations? Palaios, 20:439-451. doi:10.2110/palo.2004. P04-60

Burnham, R.J.; Pitman, N.C.A.; Johnson, K.R. \& Wilf, P. 2001. Habitat-related error estimating temperatures from leaf margins in a humid tropical forest. American Journal of Botany, 88:10961102. doi: $10.2307 / 2657093$

Cristalli, P.S. 1997. Tafoflora das Camadas Nova Iorque, depósitos Neógenos do Rio Parnaíba, Município de Nova Iorque (MA), Brasil. Programa de Pós-graduação em Geologia Sedimentar, Universidade de São Paulo, Dissertação de Mestrado, 157 p.

Cristalli, P.S. \& Bernardes-de-Oliveira, M.E.C. 1998. Tafoflora Neógena das Camadas Nova Iorque (MA-Brasil) e o seu significado paleofitogeográfico. Acta Geológica Leopoldensia, 21:55-67.

Dino, R.; Garcia, M.J.; Antonioli, L. \& Lima, M.R. 2006. Palinoflora das "Camadas Nova Iorque", Registro Sedimentar do Plioceno na Bacia do Parnaíba (Maranhão). In: SIMPÓSIO DO CRETÁCEO DO BRASIL, 7, 2006. Resumos, Serra Negra, UNESP, p. 42.

Garcia, M.J.; Bernardes-de-Oliveira, M.E.C.; Santos, M.A.; Dino, R.; Antonioli, L.; Bistrichi, C.A. \& Casado, F.C. 2007. Floras neógenas sul-americanas no contexto mundial. In: I.S. Carvalho; R.C.T. Cassab; C. Schwanke; A.C.S. Fernandes; M.A.C. Rodrigues; M.S.S. Carvalho; M. Arai \& M.E.Q. Oliveira (eds.) Paleontologia: Cenários de Vida, Interciência, p. 656-687.

Giulietti, A.M. et al. 2003. Diagnóstico da vegetação nativa do Bioma Caatinga. In: J.M.C. Silva; M. Tabarelli; M.T. Fonseca
\& L.V. Lins (eds.) Biodiversidade da caatinga: áreas e ações prioritárias para a conservação, Ministério do Meio AmbienteUniversidade Federal de Pernambuco, p. 48-78.

Greenwood, D.R. 2007. Fossil angiosperm leaves and climate: from Wolfe and Dilcher to Burnham and Wilf. Courier Forschungsinstitut Senckenberg, 258:95-108.

Guimarães, D. 1964. Geologia do Brasil. Rio de Janeiro, Departamento Nacional de Produção Mineral, 674 p.

Hinojosa, L.F.; Pérez, F.; Gaxiola, A. \& Sandoval, I. 2011. Historical and phylogenetic constraints on the incidence of entire leaf margins: insights from a new South American model. Global Ecology and Biogeography, 20:380-390. doi:10.1111/j.14668238.2010.00595.x

Hoorn, C.; Bernardes-de-Oliveira, M.E.C.; Dino, R.; Garcia, M.J.; Antonioli, L.; Casado, F.C. \& Hooghiemstra, H. 2014. Neogene climate evolution in Amazonia and Brazilian Northeast. In: I.S. Carvalho; M.J. Garcia; C.C. Lana \& O. Strohschoen Jr. (eds.) Paleontologia: Cenários de Vida-Paleoclimas, Interciência, p. 277-310.

Jacobs, B.F. \& Herendeen, P.S. 2004. Eocene dry climated and woodland vegetation in tropical Africa reconstructed from fossil leaves from northern Tanzania. Palaeogeography, Palaeoclimatology, Palaeoecology, 213:115-123. doi:10.1016/j. palaeo.2004.07.007

Kennedy, E.M.; Arens, N.C.; Reichgelt, T.; Spicer, R.A.; Spicer, T.E.V.; Stranks, L. \& Yang, J. 2014. Deriving temperature estimates from Southern Hemisphere leaves. Palaeogeography, Palaeoclimatology, Palaeoecology, 412:80-90. doi:10.1016/j. palaeo.2014.07.015

Kowalski, E.A. 2002. Mean annual temperature estimation based on leaf morphology: a test from tropical South America. Palaeogeography, Palaeoclimatology, Palaeoecology, 188:141165. doi:10.1016/S0031-0182(02)00550-3

Lima, M.R. 1991. Estudo palinológico das "Camadas Nova Iorque". Terciário do Estado do Maranhão, Brasil. In: CONGRESSO BRASILEIRO DE PALEONTOLOGIA, 12, 1991. Resumos, São Paulo, USP, p. 45.

Melo, J.D.; Silva, R.C.G.; Moura, A.A.P.; Cassab, R.C.T. \& Carvalho, M.S.S. 2005. Fósseis da localidade de Nova Iorque, Maranhão, no Acervo do Museu de Ciências da Terra/ Departamento Nacional da Produção Mineral. Anuário do Instituto de Geociências, 28:181-182.

Nimer, E. 1972. Climatologia da região Nordeste do Brasil. Introdução à climatologia dinâmica. Revista Brasileira de Geografia, 34:3-51.

Pennington, R.T.; Lavin, M.; Prado, D.E.; Pendry, C.A.; Pell, S.K. \& Butterworth, S.K. 2004. Historical climate change and speciation: neotropical seasonnaly dry forest plants show patterns of both Tertiary and Quaternary diversification. Philosophical Transactions Royal Society London Series B, 359:515-537. doi:10.1098/rstb.2003.1435

Peppe, D.J. et al. 2011. Sensitivity of leaf size and shape to climate: global patterns and paleoclimatic applications. New Phytologist, 190:724-739. doi:10.1111/j.1469-8137.2010.03615.x

Prado, D.E. 2003. As Caatingas da América do Sul. In: I.R. Leal; M. Tabarelli \& J.M.C. Silva (eds.) Ecologia e conservação da caatinga, UFPE, p. 3-74.

Raunkiaer, C. 1934. The life forms of plants and statistical plant geography. Oxford, Clarendon Press, $632 \mathrm{p}$.

Ricardi-Branco, F.S. \& Fanton, J.C.M. 2007. Principais registros paleoflorísticos do Cenozóico brasileiro. In: I.S. Carvalho; R.C.T. Cassab; C. Schwanke; A.C.S. Fernandes; M.A.C. Rodrigues; M.S.S. Carvalho; M. Arai \& M.E.Q. Oliveira (eds.) Paleontologia: Cenários de Vida, Interciência, p. 645-655. 
Rizzini, C.T. 1963. Departamento Nacional da Produção Mineral. Revista Brasileira de Geografia, 25:3-64.

Rizzini, C.T. 1979. Tratado de fitogeografia do Brasil - aspectos sociológicos eflorísticos. São Paulo, HUCITEC/EDUSP, 374 p.

Rodrigues, R.C.M. 2008. Diagnóstico do estado de conservação e das áreas de ocorrência dos recursos genéticos de abóboras (Cucurbita spp.), na agricultura familiar do Maranhão. Programa de Pós-graduação em Agroecologia, Universidade Estadual do Maranhão, Dissertação de Mestrado, 58 p.

Royer, D.L. 2012. Climate reconstruction from leaf size and shape: new developments and challenges. In: L.C. Ivany \& B.T. Huber (eds.) Reconstructing earth's deep-time climate-the state of the art in 2012, Bethesda, The Paleontological Society, p. 195-212 (Papers 18).

Santos, M.E.C.M. \& Carvalho, M.S.S. 2009. Paleontologia das bacias do Parnaíba, Grajaú e São Luís. Rio de Janeiro, CPRM, Serviço Geológico do Brasil, DGM/DIPALE, 215 p.

Velloso, A.L.; Sampaio, E.V.S.B. \& Pareyn, F.G.C. 2002. Ecorregiões propostas para o Bioma Caatinga. Recife, Associação Plantas do Nordeste, Instituto de Conservação Ambiental, The Nature Conservancy do Brasil, 76 p.
Webb, L.J. 1959. A physiognomic classification of Australian rain forest. Journal of Ecology, 47:551-570. doi:10.2307/2257290

Werneck, F.P.; Costa, G.C.; Colli, G.R.; Prado, D.E. \& Sites Jr., J.W. 2011. Revisiting the historical distribution of seasonally dry tropical forests: new insights based on palaeodistribution modelling and palynological evidence. Global Ecology and Biogeography, 20:272-288. doi:10.1111/j.14668238.2010.00596.x

Wilf, P. 1997. When are leaves good thermometers? A new case for leaf margin analysis. Paleobiology, 23:373-390. doi:10.1017/ S0094837300019746

Wilf, P.; Wing, S.L.; Greenwood, D.R. \& Greenwood, C.L. 1998. Using fossil leaves as paleoprecipitation indicators: an Eocene example. Geology, 26:203-206. doi:10.1130/00917613(1998)026<0203:UFLAPI > 2.3.CO;2

Wright, I.J. et al. 2017. Global climatic drivers of leaf size. Science, 357:917-921. doi:10.1126/science.aal4760

Received in 29 August, 2017; Accepted in 01 February, 2018. 